\title{
SOME REMARKS ON THE SPACE R2(E)
}

\section{CLAES FERNSTRÖM}

\author{
Department of Mathematics \\ University of Stockholm \\ SWEDEN
}

(Received on April 26, 1982 and in revised form on September 28, 1982)

ABSTRACT. Let $E$ be a compact subset of the complex plane. We denote by $R(E)$ the algebra consisting of the rational functions with poles off $E$. The closure of $R(E)$ in $\mathrm{L}^{\mathrm{p}}(\mathrm{E}), 1 \leq \mathrm{p}<\infty$, is denoted by $\mathrm{R}^{\mathrm{p}}(\mathrm{E})$. In this paper we consider the case $\mathrm{p}=2$. In section 2 we introduce the notion of weak bounded point evaluation of order $\beta$ and identify the existence of a weak bounded point evaluation of order $\beta, \beta>1$, as a necessary and sufficient condition for $R^{2}(E) \neq L^{2}(E)$. We also construct a compact set E such that $R^{2}(E)$ has an isolated bounded point evaluation. In section 3 we examine the smoothness properties of functions in $\mathrm{R}^{2}(\mathrm{E})$ at those points which admit bounded point evaluations.

KEY WORDS AND PHRASES. Rational functions, compact set, $L^{P}$-spaces, bounded point evaluation, weak bounded point evaluation, Bessel capacity.

1980 MATHEMATICS SUBJECT CLASSIFICATION CODES. $30 A 98$.

1. IITKODUCTION.

Let $E$ be a compact subset of the complex plane $\mathbb{C}$. For each $p, 1 \leq p<\infty$, let ${ }_{L}^{p}(E)$ be the linear space of all complex valued functions $f$ for which $|f|^{P}$ is integrable with the usual norn

$$
\left\{\int_{E}|f(z)|^{p} d m(z)\right\}^{1 / p} \text {, where m denotes the two dimensional }
$$

Lebesque measure. Denote by $R(E)$ the subspace of all rational functions having no poles on $E$ and let $R^{p}(E)$ be the closure of $R(E)$ in $L^{p}(E)$. A point $z_{0} \varepsilon$ is said to be a bounded point evaluation (BPE) for $R^{p}(E)$, if there is a constant $F$ such that 


$$
\left|f\left(z_{0}\right)\right| \leq F\left\{\int|f(z)|^{P} d m(z)\right\}^{1 / p} \text {, for all } f \in R(E) \text {. }
$$

In [1] Brennan showed that $R^{p}(E)=L^{p}(E), p \neq 2$, if and only if no point of $E$ is a $B P E$ for $\mathrm{R}^{\mathrm{P}}(\mathrm{E})$. The theorem is not true for $\mathrm{p}=2$ (See Fernströn [2] or Fernström and Polking [3].) In this paper we show that if the right hand side of (1) is made slightly larger a corresponding theorem is true for $p=2$. We also show that this theorem is best possible.

If $z_{0} \varepsilon E$ is a BPE for ${ }_{R}^{P}(E)$ there is a function $g \varepsilon L^{q}(E), \frac{1}{p}+\frac{1}{q}=1$, such that $f\left(z_{0}\right)=\int_{E} f(z) g(z) d m(z)$ for all $f \in R(E)$. The function $g$ is called a representing function for $z_{0}$. Let $B(z, \delta)$ denote the ball with radius $\delta$ and centre at $z$. We say that a set $A, A \subset \mathbb{C}$, has full area density at $z$ if $m(A \cap B(z, \delta)) m(B(z, \delta))^{-1}$ tends to one when $\delta$ tends to zero.

Suppose now that $z_{0}$ is a $B P E$ for ${ }_{R}{ }^{P}(E), 2<p$, represented by $g \in L^{q}(E)$ and $\left(z-z_{0}\right)^{-s} \phi\left(\left|z-z_{0}\right|\right)^{-1} g \in L^{q}(E)$, where $s$ is a nonnegative integer and $\phi$ is a nondecreasing function such that $r \phi(r)^{-1} y_{0}$ when $r^{\searrow_{0}}$. Then for every $\varepsilon>0$ there is a set $E_{0}$ in $E$ having full area density at $z_{0}$ such that for every $f \in R(E)$ and for all $\tau \in E_{0}$,

$$
\begin{aligned}
& \left|f(\tau)-f\left(z_{0}\right)-\frac{f^{\prime}\left(z_{0}\right)}{1 !}\left(\tau-z_{0}\right)-\ldots \frac{f^{(s)}\left(z_{0}\right)}{s !}\left(\tau-z_{0}\right)^{s}\right| \\
& \leq\left|\tau-z_{0}\right|^{s} \phi\left(\left|\tau-z_{0}\right|\right)\left\{\int_{E}|f(z)|^{p} d m(z)\right\}^{1 / p} \text {. This theorem is due to Wolf [8]. }
\end{aligned}
$$

We shall show that the theorem of Wolf is not true for $p=2$. We shall also show that a slightly weaker result is true and that this result is best possible. The main tool to show this is to construct a compact set E with exactly one bounded point derivation for $\mathrm{R}^{2}(\mathrm{E})$. A point $z_{0} \varepsilon \mathrm{E}$ is a bounded point derivation (BPD) of order $s$ for $R^{p}(E)$ if the map $f \rightarrow f(s)\left(z_{0}\right)$, $f \in R(E)$, extends from $R(E)$ to a bounded linear functional on $\mathrm{R}^{\mathrm{p}}(\mathrm{E})$.

2. BPE'S AND APPROXIMATION IN THE MEAN BY RATIONAL FUNCTIONS. Denote the Bessel kernel of order one by $G$ where $G$ is defined in terms of its Fourier transform by 


$$
\hat{\mathbf{G}}(z)=\left(1+|z|^{2}\right)^{-\frac{1}{2}} .
$$

For $\mathrm{f} \varepsilon \mathrm{L}^{2}(\mathbb{C})$ we define the potential

$$
u^{f}(z)=\int G(z-\tau) f(\tau) d n(\tau) \text {. }
$$

The Bessel capacity $C_{2}$ for an arbitrary set $X, X \subset C$, is defined by $C_{2}(x)=$ inf $\int|f(z)|^{2} \operatorname{dm}(z)$, where the infimum is taken over all $f \varepsilon L^{2}(\mathbf{C})$ such that $f(z) \geq 0$ and $\mathrm{u}^{\mathrm{f}}(\mathrm{z}) \geq 1$ for all $\mathrm{z} \varepsilon \mathrm{x}$. The set function $\mathrm{C}_{2}$ is subadditive, increasing, translation invariant and

$$
\mathrm{C}_{2}(\mathrm{~B}(\mathrm{z}, \delta)) \approx\left(\log \frac{1}{\delta}\right)^{-1}, \delta \leq \delta_{0}<1 .
$$

For further details about this capacity see Meyers [5].

The BPD's can be described by the Bessel capacity. Let $A_{n}\left(z_{0}\right)$ denote the annulus $\left\{z ; 2^{-n-1}<\left|z-z_{0}\right| \leq 2^{-n}\right\}$. The following theorem is proved in [3]:

Theorem 2.1 Let $E$ be a compact set. Then $z$ is a BPD of order $s$ for $R^{2}(E)$ if and only if

$$
\sum_{n=0}^{\infty} 2^{2 n(s+1)} c_{2}\left(A_{n}(z)-E\right)<\infty .
$$

Definition Set

$$
L_{z_{0}}(z)=\left\{\begin{array}{clll}
\log \frac{1}{\left|z-z_{0}\right|} & \text { for } & \left|z-z_{0}\right| \leq \frac{1}{e} \\
1 & \text { for } & \left|z-z_{0}\right| \geq \frac{1}{e} .
\end{array}\right\}
$$

A point $z_{0} \varepsilon E$ is called a weak bounded point evaluation (w BPE) of order $\hat{\beta}, \beta \geq 0$, for $R^{2}(E)$ if there is a constant $F$ such that

$$
\left|f\left(z_{0}\right)\right| \leq F\left\{\int_{E}|f(z)|^{2} L_{z_{0}}^{\beta}(z) d m(z)\right\}^{\frac{1}{2}}
$$

for all $f \in R(E)$.

We are now going to generalize theorem 2.1 in two directions.

Theorem 2.2 Let $s$ be a nonnegative integer and $\mathrm{E}$ a compact set. Suppose that $z_{0}$ is a $B P E$ for $R^{2}(E)$ represented by $g \varepsilon L^{2}(E)$ and that $\phi$ is a positive, nondecreasing function defined on $(0, \infty)$ such that $r \phi(r)^{-1}$ is nondecreasing and tends to zero when $r \rightarrow 0^{+}$. Then $z_{0}$ is represented by a function $g \in L^{2}(E)$ such that

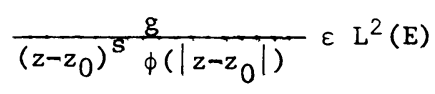




$$
\begin{aligned}
& \text { if and only if } \\
& \sum_{n=0}^{\infty} 2^{2 n(s+1)} \phi\left(2^{-n}\right)^{-2} C_{2}\left(A_{n}\left(z_{0}\right)-E\right)<\infty \text {. }
\end{aligned}
$$

Theorem 2.3 Lat $E$ be a compact set. Then $z$ is a $w$ BPE of order $\beta$ for $R^{2}(E)$ if and

$$
\sum_{n=1}^{\infty} n^{-\beta} 2{ }^{2 n} C_{2}\left(A_{n}(z)-E\right)<\infty .
$$

The proofs of theorem 2.2 and theorem 2.3 are almost the same as the proof of theorem 2.1. We omit the proofs. Wolf proved in [8] that the condition

$$
\sum_{n=0}^{\infty} 2^{2 n(s+1)} \phi\left(2^{-n}\right)^{-2} c_{2}\left(A_{n}\left(z_{0}-E\right)\right)<\infty \text { is necessary in theorem 2.2. }
$$

The compact sets $E$ for which $R^{2}(E)=L^{2}(E)$ can be described in terms of the Bessel Capacity. The following theorem is proved in Hedberg [4] and Polking [6].

Theorem 2.4 Let $\mathrm{E}$ be a compact set. Then the following are equivalent.

(i) $R^{2}(E)=L^{2}(E)$.

(ii) $C_{2}(B(z, \delta)-E)=C_{2}(B(z, \delta))$ for all balls $B(z, \delta)$.

(iii) $\lim _{\delta \rightarrow 0} \sup \frac{C_{2}(B(z, \delta)-E)}{\delta^{2}}>0$ for all $z$.

If we combine theorem 2.3 and theorem 2.4 we get the following theorem.

Theorem 2.5 Let $\beta>1$ and $E$ be a compact set. Then $L^{2}(E)=R^{2}(E)$ if and only if $E$ admits no $w$ BPE of order $\beta$ for $R^{2}(E)$.

Now we shall show that theorem 2.5 is not true for $\beta \leq 1$. We first need the following tneorem.

Theorem 2.6 There is a compact set E such that

$$
\begin{aligned}
& \text { (i) } C_{2}\left(B\left(0, \frac{1}{2}\right)-E\right)<C_{2}\left(B\left(0, \frac{1}{2}\right)\right) \\
& \text { (ii) } \sum_{n=1}^{\infty} n^{-1} 2^{2 n} C_{2}\left(A_{n}(z)-E\right)=\infty \text { for all } z
\end{aligned}
$$

The proof is a modification of a proof in [2] or [3], where a weaker theorem is proved. Since we shall need the construction of E later, we give some details.

Proof. There are constants $F_{1}$ and $F_{2}$ such that

$$
F_{1}\left(\log \frac{1}{\delta}\right)^{-1} \leq C_{2}(B(z, \delta)) \leq F_{2}\left(\log \frac{1}{\delta}\right)^{-1} \quad \text { for all } \delta, \delta \leq \delta_{0}<1
$$


Choose $\alpha, \alpha \geq 1$, such that

$$
\frac{F_{2}}{\alpha} \sum_{n=2}^{\infty} \frac{1}{n \log ^{2} n}<C_{2}\left(B\left(0, \frac{1}{2}\right)\right) \text {. }
$$

Let $A_{0}$ be the closed unit square with centre at the origin. Cover $A_{0}$ with $4^{n}$ squares with side $2^{-n} \cdot$ Call the squares $A_{n}{ }^{(i)}, i=1,2, \ldots, 4^{n}$. In every $A_{n}$ (i) put an open disc $B_{n}{ }^{(i)}$ such that $B_{n}{ }^{(i)}$ and $A_{n}{ }^{(i)}$ have the same centre and the radius of $B_{n}$ (i) is $\exp \left(-\alpha 4^{n} n \log n\right)$. Repeat the construction for all $n, n \geq 2$.

Set

$$
E=A_{0}-\bigcup_{n=2}^{\infty} \bigcup_{i=1}^{4^{n}} B_{n}(i)
$$

The subadditivity of $\mathrm{C}_{2}$ now gives (i).

In order to prove (ii) it is enough to prove

$$
\mathrm{C}_{2}\left(\mathrm{~A}_{\mathrm{n}}{ }^{(i)}-\mathrm{E}\right) \geq \frac{\mathrm{F}_{1}}{32 \alpha 4^{\mathrm{n}} \log \mathrm{n}} \text { for } \mathrm{a} 11 \mathrm{n}, \mathrm{n} \geq \mathrm{n}_{0} \text {. }
$$

Consider all $\mathrm{B}_{\mathrm{k}}^{(\mathrm{i})}, \mathrm{n} \leq \mathrm{k} \leq \mathrm{n}^{2}$, such that $\mathrm{B}_{\mathrm{k}}{ }^{(\mathrm{i})} \subset \mathrm{A}_{\mathrm{n}}{ }^{\text {(i) }}$.

We get $4^{\ell}$ discs with radius $\exp \left(-\alpha 4^{n+l}(n+l) \log ^{2}(n+l)\right), 0 \leq \ell \leq n^{2}-n$.

Call the discs

$$
D_{n}(r), r=1,2, \ldots, \frac{4^{n^{2}-n+1}-1}{3} \text {. }
$$

Thus

$$
\begin{gathered}
\frac{F_{1}}{\alpha 4^{n}} \sum_{j=n}^{n^{2}} \frac{1}{j \log ^{2} j} \leq \sum_{r} C_{2}\left(D_{n}{ }^{(r)}\right) \leq \frac{F_{2}}{\alpha 4^{n}} \sum_{j=n}^{n^{2}} \frac{1}{j \log ^{2} j} . \\
\quad \text { Set } D_{n}=\bigcup_{r} D_{n}{ }^{(r)} .
\end{gathered}
$$

Since the distances between the discs are large compared to their radii, it can be shown that

$$
\mathrm{C}_{2}\left(\mathrm{D}_{\mathrm{n}}\right) \geq \frac{1}{8} \sum_{\mathrm{r}} \mathrm{C}_{2}\left(\mathrm{D}_{\mathrm{n}}{ }^{(\mathrm{r})}\right) \text {, if } \mathrm{n} \text { is large. }
$$

(See theorem $2^{\prime}$ in [2] or theorem 2 in [3] for a proof.)

Thus if $\mathrm{n}$ is large,

$$
C_{2}\left(A_{n}(i)-E\right) \geq C_{2}\left(D_{n}\right) \geq \frac{F_{1}}{8 \alpha 4^{n}} \sum_{j=n}^{n^{2}} \frac{1}{j \log ^{2} j} \geq \frac{F_{1}}{16 \alpha 4^{n} \log n} \text {, }
$$

which is $(2.1)$

$$
q \cdot e \cdot d \text {. }
$$


Theorem 2.7 There is a compact set E such that
(i) $L^{2}(E)=R^{2}(E)$
(ii) E has no $w$ BPE of order one for $R^{2}(E)$.

Proof The theorem follows immediately from theorem 2.3, 2.4, and 2.6.

3. BPE'S AND SHOOTHNESS PROPERTIES OF FUNCTIONS IN $\mathrm{R}^{2}(\mathrm{E})$.

In this section we treat the theorem of Wolf mentioned in the introduction for the case $p=2$.

Theorem 3.1 Let $\phi$ be a positive, nondecreasing function defined on $(0, \infty)$ such that $\mathrm{rL}_{0}(\mathrm{r}) \phi(\mathrm{r})^{-1}$ is nondecreasing and $\mathrm{rL}_{0}(\mathrm{r}) \phi(\mathrm{r})^{-1} \rightarrow 0$ when $\mathrm{r} \rightarrow 0^{+}$. Suppose that $z_{0}$ is a $B P E$ for $R^{2}(E)$ represented by $g$ and $\left(z-z_{0}\right)^{-s} \phi\left(\left|z-z_{0}\right|\right)^{-1} g \in L^{2}(E)$, where $s$ is a nonnegative integer. Then for every $\beta>1$ and $\varepsilon>0$ there is a set $E_{0}$ in $E$, having full area density at $z_{0}$, such that for every $f \varepsilon R(E)$ and every $\tau \in E_{0}$

$$
\begin{aligned}
& \left|f(\tau)-f\left(z_{0}\right)-\frac{f^{\prime}\left(z_{0}\right)}{1 !}\left(\tau-z_{0}\right)-\ldots-\frac{f^{(s)}\left(z_{0}\right)}{s !}\left(\tau-z_{0}\right)\right| \\
& \leq \varepsilon\left|\tau-z_{0}\right|^{s} \phi\left(\left|\tau-z_{0}\right|\right) \quad\left\{\int_{E}|f(z)|^{2} L_{z_{0}}^{\beta}(z)\right\}^{1 \frac{1}{2}} .
\end{aligned}
$$

The proof of theorem 3.1 is only a minor modification of the proof of theorem 4.1 in [3]. lioreover, there is a proof of theorem 3.1 for $\beta=2$ in Wolf [7]. We omit the proof.

Remark. Let $z_{0} \in \partial E$ (the boundary of $E$ ) be both a BPE for $K^{2}(E)$ and the vertex of a sector contained in Int $E$. Let $L$ be a line which passes through $z_{0}$ and bisects the secotr. Let $\varepsilon>0$ and let $\phi$ be as in theorem 2.2. For those $y \varepsilon L \cap E$ that are sufficiently near $z_{0}$ Wolf showed in [9] that

$$
\left|f(y)-f\left(z_{0}\right)\right| \leq \varepsilon \phi\left(\left|y-z_{0}\right|\right) \quad\left\{\int|f(z)|^{2} d m(z)\right\}^{\frac{1}{2}} \quad \text { for all } f \varepsilon R(E) .
$$

Our next step is to prove that theorem 3.1 is not true for $\beta=1$. We first need a theorem, which we think is interesting in itself.

Theorem 3.2 Let $\mathrm{s}$ be a nonnegative integer. Then there is a compact set $\mathrm{E}$ such that

$$
\begin{aligned}
& \text { (i) } \sum_{i=1}^{\infty} n^{-1} 2^{2 n} C_{2}\left(A_{n}(z)-E\right)=\infty \text { if } z \neq 0 . \\
& \text { (ii) } \sum_{n=1}^{\infty} 2^{2 n(s+1)} C_{2}\left(A_{n}(0)-E\right)<\infty .
\end{aligned}
$$


Proof We shall modify the set constructed in the proof of theorem 2.6. Let $B_{j}^{(k)}$ denote the same discs as in that proof. Let all $\mathrm{B}_{j}^{(k)}$ which intersect $\mathrm{A}_{1}(0)$ be denoted by $A_{11}, A_{12}, A_{13}, \ldots$ so that their diameters are decreasing. Choose $j_{1}$ so that

$$
2^{2(s+1)} \sum_{j>j_{1}} C_{2}\left(A_{1 j}\right)<2^{-1}
$$

and

$$
\operatorname{diam}\left(A_{1 j_{1}}\right)<2^{-3} \text {. }
$$

Suppose that we have chosen $j_{1}, \ldots j_{n}$. Let all $B_{j}^{(k)}$ which intersect $A_{n+1}(0)$ and which do not coincide with $A_{11}, \ldots, A_{1 j_{1}}, \ldots, A_{n 1}, \ldots, A_{n j_{n}}$, be denoted by $A_{n+1}, A_{n+2}, A_{n+3}, \ldots$ so that their diameters are decreasing.

Choose $j_{n+1}$ so that

$$
2^{2(n+1)(\Omega+1)} \sum_{j>j_{n+1}} C_{2}\left(A_{n+1} j\right)<2^{-(n+1)}
$$

and $\operatorname{diam}\left(A_{n+1} j_{n+1}\right)<2^{-(n+3)}$.

Let $A_{0}$ be the closed unit square with centre at the origin. Set $E=A_{0}-$ (The union of all $B_{j}^{(k)}$ such that $B_{j}^{(k)} \neq A_{n m}, 1 \leq n<\infty$ and $\left.1 \leq m \leq j_{n}\right)$.

We have

$$
\sum_{n=1}^{\infty} 2^{2 n(s+1)} C_{2}\left(A_{n}(0)-E\right) \leq \sum_{n=1}^{\infty} 2^{-n}<\infty .
$$

Let $z \neq 0$. If $\ell$ is large all $B_{j}{ }^{(k)}, B_{j}^{(k)} \subset A_{\ell}(z)$, differ fro $A_{n m}$, $1 \leq \mathrm{n}<\infty$ and $1 \leq \mathrm{m} \leq \mathrm{j}_{\mathrm{n}}$ Now exactly as in proof of theorem 2.6 it follows

$$
\sum_{n=1}^{c} n^{-1} 2^{2 n} c_{2}\left(A_{n}(z)-E_{1}\right)=\infty
$$

$$
\text { q.e.d. }
$$

Corollary 3.3 There is a compact set E with exactly one BPD of order $s$ for $R^{2}(E)$.

Proof Just combine theorem 3.2 and 2.1 .

Remark The situation for $p \neq 2$ is different. In [1] Brennan showed that if almost all points $z \in E, E$ compact, are not BPE for $R^{P}(E), E$ admits no BPE's for $R^{2}(E)$.

Theorem 3.4 Let $s$ be a nonnegative integer and $\phi$ be as in theorem 2.2. Then there is a compact set $\mathrm{E}$ such that

(i) $z_{0}$ is a BPE for $R^{2}(E)$.

(ii) There is a representing function $g$ for $z_{0}$ that satisfies 


$$
\left(z-z_{0}\right)^{-s} \phi\left(\left|z-z_{0}\right|\right)^{-1} g \in L^{2}(E) \text {. }
$$

(iii) For every $\tau \varepsilon E, \tau \neq z_{0}$, and every positive integer $n$ there is a function $f \varepsilon R(E)$ such that

$$
\begin{aligned}
& \left|f(\tau)-f\left(z_{0}\right)-\frac{f^{\prime}\left(z_{0}\right)}{1 !}\left(\tau-z_{0}\right)-\ldots-\frac{f^{(s)}\left(z_{0}\right)}{s !}\left(\tau-z_{0}\right)\right|> \\
& >n\left\{\int|f(z)|^{2} L_{E}(z) d m(z)\right\}^{\frac{1}{2}} .
\end{aligned}
$$

Proof Theorem 3.2 gives that there is a compact set $E$ such that

$$
\begin{aligned}
& \sum_{n=1}^{\infty} n^{-1} 2^{2 n} c_{2}\left(A_{n}(z)-E\right)=\infty, z \neq z_{0} \\
& \sum_{n=1}^{\infty} 2^{2 n(s+1)} \phi\left(2^{-n}\right)^{-2} c_{2}\left(A_{n}\left(z_{0}\right)-E\right)<\infty .
\end{aligned}
$$

Now theorem 2.1 gives (i) and theorem 2.2 gives (ii). Moreover theorem 2.1 gives that $z_{0}$ is a BPD of order $s$ for $R^{2}(E)$ and theorem 2.3 that $\tau$ is not a $w$ BPE of order 1 for $\mathrm{R}^{2}(\mathrm{E})$. This gives (iii).

\section{REFERENCES}

1. Brennan, J. E., Invariant Subspaces and Rational Approximation, J. Functional Analysis, $\underline{7}(1971), 285-310$.

2. Fernström, C., Bounded Point Evaluations and Approximation in $\mathrm{L}^{\mathrm{p}}$ by Analytic Functions, in "Spaces of Analytic Functions Kristiansand, Norway 1975", 65-68, Lecture Notes in Mathematics No 512, Springer-Verlag, Berlin 1976.

3. Fernström, C and Polking, J. C., Boundee Point Evaluations and Approximation in $\mathrm{L}^{\mathrm{P}}$ by Solutions of Elliptic Partial Differential Equations. J. Functional Analysis, 28, 1-20 (1978).

4. Hedberg, L. I., Non Linear Potentials and Approximation in the Mean by Analytic Functions, Math. Z., 129 (1972) 299-319.

5. Meyers, N. G., A Theory of Capacities for Potentials of Functions in Lebesgue Classes, Math. Scand., 26 (1970), 255-292.

6. Polking, J. C. Approximation in $\mathrm{L}^{\mathrm{P}}$ by Solutions of Elliptic Partial Differential Equations, Amer. J. Math., 94 (1972), 1231-1244.

7. Wolf, E., Bounded Point Evalutions and Smoothness Properties of Functions in ${ }_{\mathrm{R}}^{\mathrm{P}}(\mathrm{X})$, Doctoral Dissertation, Brown University, Providence, R.I., 1976.

8. Wolf, E., Bounded Point Evaluations and Smoothness Properties of Functions in $\mathrm{R}^{\mathrm{P}}(\mathrm{X})$, Trans. Amer. Math. Soc. 238 (1978), 71-88.

9. Wolf, E., Smoothness Properties of Functions in $R^{2}(X)$ at Certain Boundary Points, Internat. J. Math. and Math. Sci., 2 (1979), 415-426. 


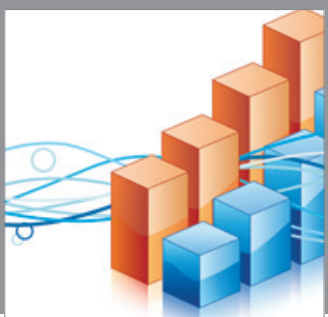

Advances in

Operations Research

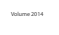

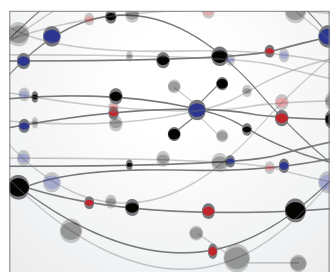

\section{The Scientific} World Journal
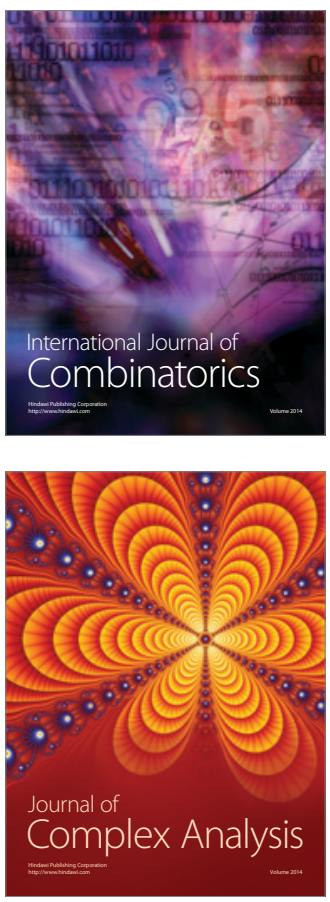

International Journal of

Mathematics and

Mathematical

Sciences
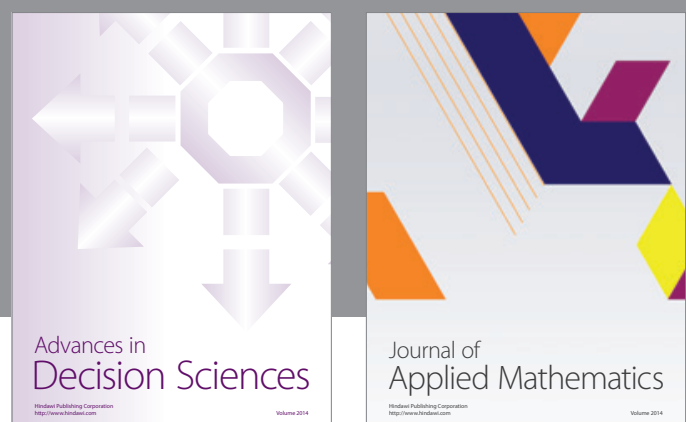

Journal of

Applied Mathematics
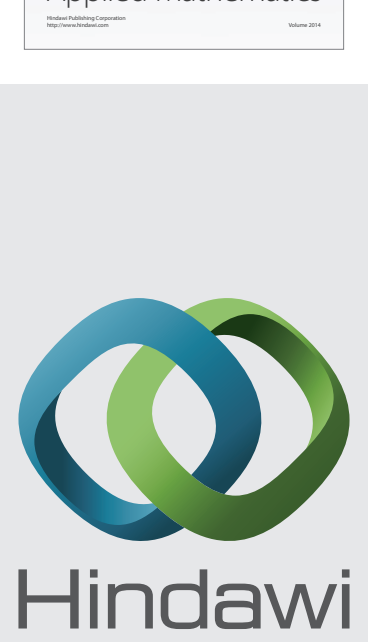

Submit your manuscripts at http://www.hindawi.com
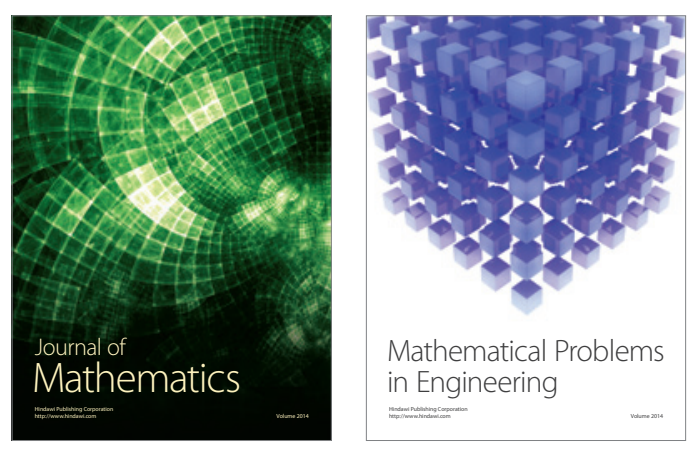

Mathematical Problems in Engineering
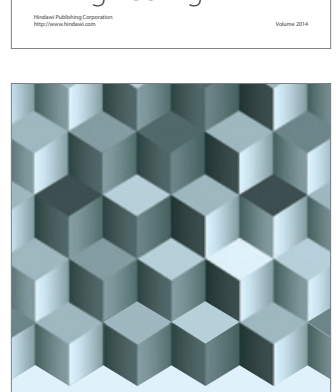

Journal of

Function Spaces
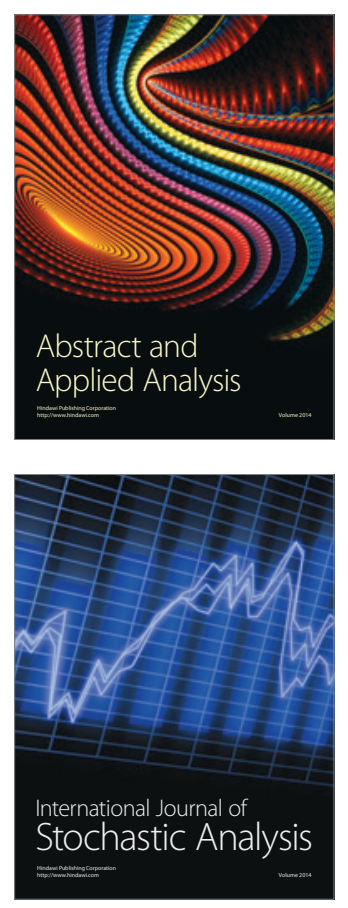

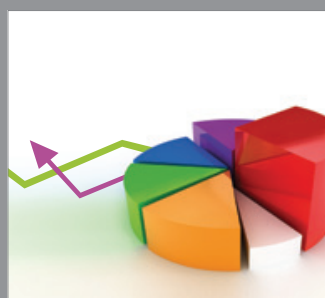

ournal of

Probability and Statistics

Promensencen
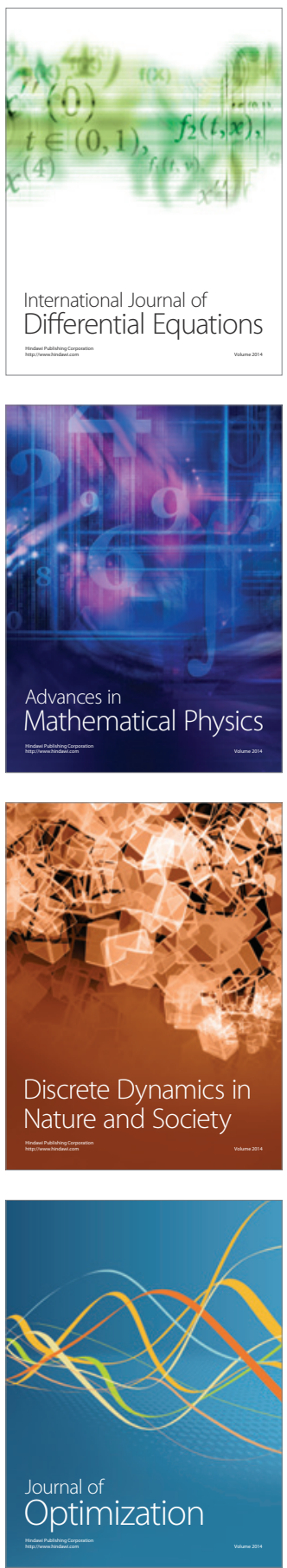